\title{
Narrow resonances and black-hole-like absorption in a non-black-hole metric
}

\author{
V. V. Flambaum and G. H. Gossel \\ School of Physics, University of New South Wales, Sydney 2052, Australia \\ G. F. Gribakin \\ Department of Applied Mathematics and Theoretical Physics, \\ Queen's University, Belfast BT7 $1 N N$, Northern Ireland, UK
}

(Dated: November 14, 2017)

\begin{abstract}
A massive body with the Schwarzschild interior metric (perfect fluid of constant density) develops a pressure singularity at the origin when the radius of the body $R$ approaches $9 r_{s} / 8$, where $r_{s}$ is the Schwarzschild radius. We show that a quantum scalar particle scattered in this gravitational field possesses a dense spectrum of narrow resonances. Their density and lifetimes tend to infinity in the limit $R \rightarrow 9 r_{s} / 8$, and we determine the cross section of the particle capture into these quasibound states. Therefore, a body that is not a black hole demonstrates black-hole-like absorption.

PACS numbers: 04.62.+v, 04.70.Dy, 04.70.-s
\end{abstract}

\section{INTRODUCTION}

We found in our previous work [1] that the scattering of a massless scalar particle in a near-black-hole metric of a massive body whose radius $R$ slightly exceeds the Schwarzschild radius $r_{s}$, is characterized by a dense spectrum of resonances. For $R \rightarrow r_{s}$ both the resonance energy spacing $D$ and their width $\gamma$ tend to zero, while their ratio remains finite $\left(\gamma / D \simeq 2 \varepsilon^{2} r_{s}^{2} / \pi\right)$ and tends to zero for small energies $\varepsilon$. (We use units where $\hbar=c=1$.) This allowed us to define the cross section for particle capture into these long-lived states in the spirit of the optical model [2], by averaging over a small energy interval containing many resonances. Note that this capture emerges in a purely potential scattering problem, without any absorption introduced $a$ priori. Somewhat unexpectedly, the capture cross section turned out to be equal to the cross section obtained by assuming total absorption at the event horizon.

In particular, in the zero-energy limit our result coincides with Unruh's absorption cross section $\sigma_{a}=4 \pi r_{s}^{2}$ for a black hole [3 9]. This shows that a non-singular static metric can acquire black-hole properties prior to the actual formation of the black hole.

It is interesting to see whether this resonance absorption phenomenon is specific to near-black-hole metrics, or if there are other instances of similar behaviour. For example, consider a massive star modelled as an incompressible fluid sphere. The interior of such a body is described by the Schwarzschild interior metric [10]. This metric develops a pressure singularity at the origin for $R \rightarrow 9 r_{s} / 8$, far form the black-hole limit. In Ref. [11] we examined the spectrum of discrete bound states for a massive scalar particle in the gravitational field described by this metric. It was found that near the singularity this spectrum acquires some black-hole-like features. In particular, all the levels with finite principal quantum number $n$ collapse towards zero energy (binding energy is $-m c^{2}$ ), i.e., the spectral density becomes infinite, similar to that of a near-black-hole metric (cf. Ref. [12]).
This gives us a motivation to search for narrow resonances in the Schwarzschild interior metric. Indeed, in the present work we find such resonances in the limit $R \rightarrow 9 r_{s} / 8$. However, in contrast to the black-hole-like case considered in Ref. [1], the effective potential produced by the Schwarzschild interior metric possesses a barrier near the boundary, and the long resonance lifetimes are due to the particle having to tunnel through this barrier to the surface of the body.

Similar to the black-hole-like behaviour for $R \rightarrow r_{s}$, the resonance width $\gamma$ and energy spacing $D$ tend to zero for the Schwarzschild interior metric in the limit $R \rightarrow 9 r_{s} / 8$, while their ratio $\gamma / D$ remains finite and tends to zero at small energies $\varepsilon$. One can thus define the "optical" capture cross section by averaging over a small energy interval containing many resonances. In contrast to the near-black-hole case, where the cross section remains finite, the absorption cross section for the Schwarzschild interior metric tends to zero at zero energy. However, this metric still possesses black-hole-like absorption for nonzero energies. This means that such an object may gravitationally absorb particles in a black-hole-like manner despite the fact that its metric never approaches that of a black hole.

In what follows we analyse the massless scalar particle scattering problem for the Schwarzschild interior metric both numerically and analytically. Although the latter treatment of the problem is only approximate, it confirms all the important features of the emerging resonant scattering picture.

\section{RADIAL KLEIN-GORDON EQUATION}

The Klein-Gordon equation for a scalar particle of mass $m$ in a curved space-time with the metric $g_{\mu \nu}$ is

$$
\partial_{\mu}\left(\sqrt{-g} g^{\mu \nu} \partial_{\nu} \Psi\right)+\sqrt{-g} m^{2} \Psi=0 .
$$

For a particle of energy $\varepsilon$ in a spherically symmetric field we seek solution of Eq. (1) in the form $\Psi(x)=$ 
$e^{-i \varepsilon t} \psi(r) Y_{l m}(\theta, \varphi)$. Considering for simplicity the case of a massless particle with zero angular momentum $(l=0)$ and the metric of the form

$$
\mathrm{d} s^{2}=a(r) d t^{2}-b(r) d r^{2}-r^{2} d \Omega^{2},
$$

where $d \Omega^{2}=d \theta^{2}+\sin ^{2} \theta d \varphi^{2}$, the radial wave equation is given by

$$
\psi^{\prime \prime}(r)+\left[\frac{2}{r}+\frac{h^{\prime}(r)}{h(r)}\right] \psi^{\prime}(r)+\frac{\varepsilon^{2}}{h^{2}(r)} \psi(r)=0
$$

where $h(r)=\sqrt{a(r) / b(r)}$. By transforming the radial function as $\psi(r)=r^{-1} \phi(r) / \sqrt{h(r)}$, Eq. (3) can be cast in the following Schrödinger-like form,

$$
\phi^{\prime \prime}(r)+\left\{\frac{\varepsilon^{2}}{h^{2}}+\frac{1}{4}\left[\frac{h^{\prime}}{h}\right]^{2}-\frac{h^{\prime \prime}}{2 h}-\frac{h^{\prime}}{r h}\right\} \phi(r)=0 .
$$

This equation is convenient for deriving its semiclassical (WKB) solution. For small $h(r)$ (near $r=0$, see below) the first term in braces dominates and this solution is

$$
\phi(r)=\sqrt{\frac{h(r)}{\varepsilon}} \sin \left(\varepsilon \int_{0}^{r} \frac{d r}{h(r)}\right) .
$$

In fact, it is easy to verify that this solution is exact if the $-h^{\prime} / r h$ term in braces in Eq. (4) is neglected.

\section{PHASESHIFT FOR THE SCHWARZSCHILD INTERIOR METRIC}

Outside a spherically symmetric, non-rotating body of mass $M$ and radius $R$ the metric is given by the Schwarzschild solution

$$
d s^{2}=\left(1-\frac{r_{s}}{r}\right) d t^{2}-\left(1-\frac{r_{s}}{r}\right)^{-1} d r^{2}-r^{2} d \Omega^{2},
$$

where $r_{s}=2 G M$ is the Schwarzschild radius of the body and $G$ is the gravitational constant. Hence, for $r>R$ Eq. (3) takes the form

$$
\psi^{\prime \prime}(r)+\left(\frac{1}{r-r_{s}}+\frac{1}{r}\right) \psi^{\prime}(r)+\frac{r^{2} \varepsilon^{2}}{\left(r-r_{s}\right)^{2}} \psi(r)=0 .
$$

The Schwarzschild interior solution describes a static spherical mass of incompressible perfect fluid with constant density, and is given by Eq. (2) with

$$
\begin{aligned}
& a(r)=\left(\frac{3}{2} \sqrt{1-\frac{r_{s}}{R}}-\frac{1}{2} \sqrt{1-\frac{r_{s} r^{2}}{R^{3}}}\right)^{2}, \\
& b(r)=\left(1-\frac{r_{s} r^{2}}{R^{3}}\right)^{-1},
\end{aligned}
$$

so that

$$
h(r)=\frac{1}{2} \sqrt{1-\frac{r_{s} r^{2}}{R^{3}}}\left(3 \sqrt{1-\frac{r_{s}}{R}}-\sqrt{1-\frac{r_{s} r^{2}}{R^{3}}}\right) .
$$

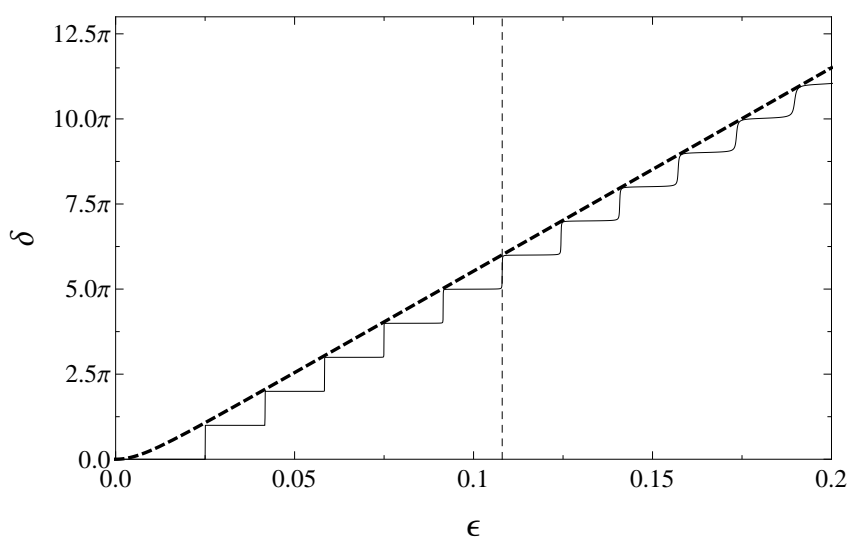

FIG. 1: The short-range phaseshift $\delta$ obtained numerically as a function of $\epsilon=\varepsilon R$ (solid line) and the semiclassical phase, Eq. (13), at the classical turning point (thick dashed line) for $\xi=8 / 9-r_{s} / R=0.00014$. The vertical dashed line corresponds to the sixth resonance at $\epsilon \approx 0.1080$.

This metric is valid for $r_{s}<8 R / 9$ but develops a singularity as $a(0)$ vanishes for $r_{s}=8 R / 9$ [13].

Using $h(r)$ from Eq. (8) in Eq. (3) gives the radial wave equation for $r<R$. For $r_{s}<8 R / 9$ this metric is smooth at the origin. Hence, the regular solution for $l=0$ must satisfy the boundary conditions $\psi^{\prime}(0)=0, \psi(0) \neq 0$. The value of $\psi(0)$ only affects the normalization of the wavefunction and we set $\psi(0)=1$. We solve the interior equation numerically using Mathematica [14].

This solution supplies the boundary condition for the exterior wavefunction at the surface of the body $r=R$. ( $R=1$ is used in the numerical calculations.) Equation (7) is then integrated outwards to large distances $r \gg r_{s}$. In this asymptotic region Eq. (7) takes the form of the nonrelativistic Shrödinger equation for a particle with momentum $\varepsilon$ and unit mass in the attractive Coulomb potential with charge $Z=-r_{s} \varepsilon^{2}$. Here the wavefunction can be matched with the asymptotic Coulomb solution [2],

$$
\psi(r) \propto \sin \left[\varepsilon r-(Z / \varepsilon) \ln 2 \varepsilon r+\delta_{C}+\delta\right],
$$

where $\delta_{C}=\arg \Gamma(1+i Z / \varepsilon)$ is the Coulomb phaseshift, to find the short-range phaseshift $\delta$. The latter is determined almost exclusively by the interior metric, and carries important information about the behaviour of the wavefunction at $r<R$.

Unlike $\delta_{C}$ which is small and has a weak dependence on the energy, the phaseshift $\delta$ becomes large when $r_{s}$ approaches $8 R / 9$, i.e., for

$$
\xi \equiv 8 / 9-r_{s} / R \ll 1 .
$$

This phaseshift also has a strong dependence on the energy of the particle, as shown by the solid line in Fig. 1. Similar to the case of a near-black-hole metric considered in Ref. [1], this phaseshift goes through many steps 


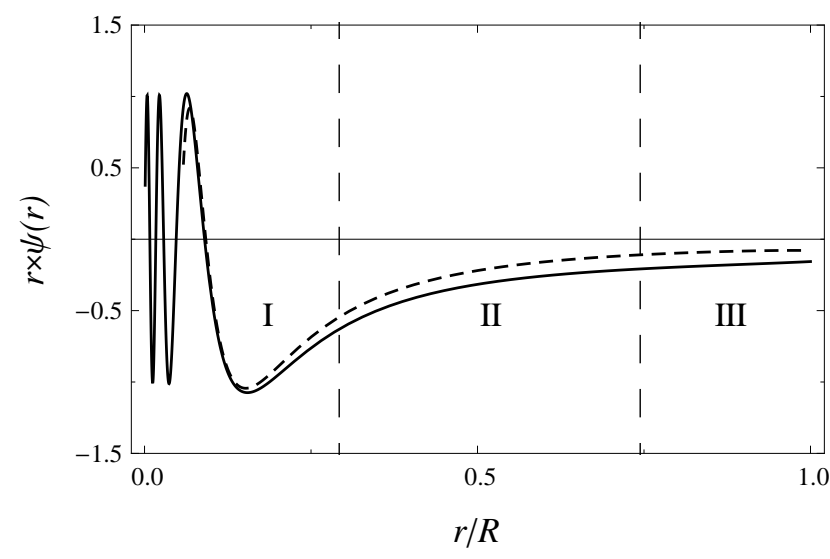

FIG. 2: The radial wavefunction at the energy $\epsilon=0.1080$ of the sixth resonance for $\xi=0.00014$ (solid curve). The dashed curve is the analytical approximation for the wavefunction given by Eqn. (16) for the energy $\varepsilon_{n}(n=6)$ from Eqn. (19). The vertical lines indicate the two classical turning points defined by $p(r)=0$. Regions I and III are classically allowed, while II is classically forbidden.

of the size $\pi$. Each of these steps corresponds to a resonance, i.e., a long-lived quasibound states of the projectile and the target. For energies corresponding to the midpoints of the steps (where the derivative $d \delta / d \varepsilon$ is largest) the magnitude of the wavefunction $\psi(r)$ inside the body $(r<R)$ is much greater than outside. This is a signature of a quasibound state. The wavefunction at one of the resonances is shown in Fig. 2 ,

The resonances observed in the phaseshift $\delta$ are charterised by their energies $\varepsilon_{n}$ and widths $\gamma_{n}$, which can be determined numerically by fitting the "steps" in $\delta$ by $\arctan \left[2\left(\varepsilon-\varepsilon_{n}\right) / \gamma_{n}\right]+$ const. [2], or analytically (see Sec. [V). We show below (see Figs. 3 and [4) that as the Schwarzschild radius $r_{s}$ tends to $8 R / 9$, i.e., for $\xi \rightarrow 0$, both the energies and the widths of the resonances tend to zero (i.e., their lifetimes tend to infinity). This means that in this limit the massive body with the Schwarzschild interior metric develops absorption properties usually typical of black holes.

\section{ENERGIES AND WIDTHS OF THE RESONANCES}

\section{A. Schwarzschild interior}

When the wave equation is written in the Schrödingerlike form (4), the coefficient in braces playes the role of an effective potential for the motion a particle with the classical momentum

$$
p(r)=\left\{\frac{\varepsilon^{2}}{h^{2}}+\frac{1}{4}\left[\frac{h^{\prime}}{h}\right]^{2}-\frac{h^{\prime \prime}}{2 h}-\frac{h^{\prime}}{r h}\right\}^{1 / 2} .
$$

In the case of the Schwarzschild interior metric, $h(r)$ is given by Eq. (8). For $\xi \ll 1$ and $r \ll R$ (in practice $r<$ $0.5 R$ is sufficient) $h(r)$ can be approximated as follows,

$$
h(r) \simeq \frac{9 \xi}{4}+\frac{2 r^{2}}{9 R^{2}}-\frac{4 r^{4}}{81 R^{4}} .
$$

This shows that for small $r$, where the first term in braces in Eq. (11) dominates, the particle has a large classical momentum (e.g., $p(0) \simeq 4 \varepsilon / 9 \xi$ ). This corresponds to the motion in a deep classically allowed region near the origin. Analysis of Eqs. (8) and (11) shows that for $\xi \ll 1$ there is a second classically allowed region near the boundary with a broad, nonsemiclassical potential barrier in between, cf. Fig. 2. These regions are separated by two classical turning points $r_{1}$ and $r_{2}$ given by the roots of $p(r)=0$.

The above picture explains the origins of the dense spectrum of narrow resonances observed in the limit $\xi \rightarrow 0$. To estimate the resonance widths analytically we require an expression for the scattering matrix, which is constructed using the procedure outlined in the following subsections.

\section{Region I: origin to barrier}

In this region $\left(0 \leq r \ll r_{1}\right)$ the $\varepsilon^{2} / h^{2}$ term in Eq. (11) dominates near the origin for $\xi \ll 1$. Dropping the last term in Eq. (12) we obtain the semiclassical phase of the wavefunction $\phi(r)$, Eq. (5),

$$
\begin{aligned}
\int_{0}^{r} \frac{\varepsilon}{h(r)} d r & \simeq \varepsilon \int_{0}^{r}\left(\frac{9 \xi}{4}+\frac{2 r^{2}}{9 R^{2}}\right)^{-1} d r \\
& =\frac{\epsilon \sqrt{2}}{\sqrt{\xi}} \arctan \left(\frac{\rho \sqrt{8}}{9 \sqrt{\xi}}\right),
\end{aligned}
$$

where $\epsilon=\varepsilon R$ and $\rho=r / R$ are the scaled energy and radial coordinate, respectively. The wavefunction in this region is then

$$
\phi_{\mathrm{I}}(\rho)=C \sqrt{\frac{9 \xi}{4}+\frac{2 \rho^{2}}{9}} \sin \left[\frac{\epsilon \sqrt{2}}{\sqrt{\xi}} \arctan \left(\frac{\rho \sqrt{8}}{9 \sqrt{\xi}}\right)\right],
$$

where $C$ depends on normalization.

\section{Region II: suppression by barrier}

In the intermediate range, $\sqrt{\xi} \ll \rho \lesssim 0.5$, the square of the classical momentum (11) simplifies to

$$
p^{2}(r) \simeq \frac{81 R^{4} \varepsilon^{2}}{4 r^{4}}-\frac{2}{r^{2}} .
$$

In this case Eq. (4) has an exact analytical solution,

$$
\phi_{\mathrm{II}}(\rho)=A \rho \sqrt{81 \epsilon^{2}+4 \rho^{2}} \sin \left[\Phi+\frac{9 \epsilon}{2 \rho}+\arctan \left(\frac{2 \rho}{9 \epsilon}\right)\right],
$$




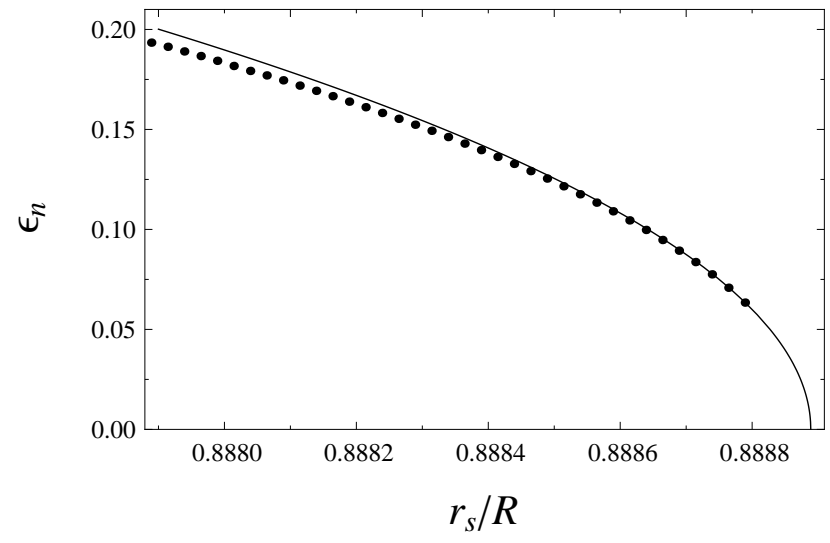

FIG. 3: Energies of the $n=4$ resonance given by Eq. (19) (solid line) and obtained numerically by fitting the resonance jumps of the phaseshift $\delta$ (solid circles) as a function of $r_{s} / R$.

where $\Phi$ and $A$ are constants determined by matching to the solution (14) to the left of the first classical turning point $\rho_{1}=9 \epsilon / \sqrt{8}$ [from Eq. (15)].

For $\sqrt{\xi} \ll \rho<\rho_{1}$ both Eq. (14) and Eq. (16) are valid (the former is valid at the origin while the latter is not). Matching is done by first expanding the arctan term in Eq. (14) for $\rho / \sqrt{\xi} \gg 1$, and neglecting the arctan term in Eq. (16), which is justified for $2 \rho / 9 \epsilon \ll 1$. This yields the phase

$$
\Phi=-\frac{\pi \epsilon}{\sqrt{2 \xi}}
$$

When the energy is on resonance, the wavefunction decreases under the barrier (Region II in Fig. 2). Considering Eq. (16) for $\rho \gg \rho_{1}$ (but keeping $\rho<0.5$, which is possible for low energies $\epsilon \ll 1$ ), and expanding the arctan function for large arguments, we have

$$
\phi_{\mathrm{II}}(\rho) \simeq-2 A \rho^{2} \sin \left[\frac{\pi \epsilon}{\sqrt{2 \xi}}-\frac{\pi}{2}-\frac{1}{3}\left(\frac{9 \epsilon}{2 \rho}\right)^{3}\right] .
$$

This function represents a decreasing solution $\phi_{\mathrm{II}}(\rho) \propto$ $\rho^{-1}$ only if we require $\pi \epsilon / \sqrt{2 \xi}-\pi / 2=n \pi(n=1,2, \ldots)$, which gives the resonance energies

$$
\epsilon_{n}=\sqrt{2 \xi}(n+1 / 2) .
$$

Hence the resonances form an equispaced spectrum similar to that of a harmonic oscillator (starting from $n=1$ though). This is in agreement with the behaviour of the phaseshift in Fig. 1.

According to Eq. (19), for $\xi \rightarrow 0$ the energies of all resonances tend to zero. This behaviour is shown in Fig. 3 which compares the analytical expression for $\epsilon_{n}$ with the values obtained numerically from the phaseshift $\delta$.

\section{Region III: barrier to boundary}

To calculate the $S$-matrix and determine the resonance widths analytically we need to match the interior and exterior solutions at the boundary $r=R$. Formally, the effective potential in Eq. (4) contains a second classical turning point $r_{2}$, which defines the outer classically allowed region, $r_{2}<r<R$. In this region the classical momentum remains small and the wavefunction changes little between $r_{2}$ and $R$ (see Fig. 2). Indeed, it is easy to check that the classical momentum takes its largest values at the boundary $r=R$, and for $\xi \rightarrow 0$ one has $p(R)=\sqrt{81 \varepsilon^{2}+58 R^{-2}}$. This momentum is much smaller than the momenta at the origin (see Sec. IV A 1) or at small $r$ [see Eq. (15)].

Therefore in generating an approximate analytical solution over the entire interior region we assume that the major contributions arise from regions I and II. Changes in the wavefunction due to the potential in region III give rise to higher-order effects which we calculate as corrections at the end of this section. Thus we formally extend the solution in Eq. (16) to the boundary $\rho=1$ where it is matched to an appropriate solution of the exterior equation as discussed below.

\section{B. Schwarzchild exterior solution}

For low-energy scattering, $\varepsilon R \ll 1$, the last term in the exterior equation (77) can be neglected near the boundary. Hence, for $r \sim R$, the solution of Eq. (7) is

$$
\psi(r)=\alpha \ln \left(\frac{r-r_{s}}{r}\right)+\beta
$$

where $\alpha$ and $\beta$ are constants determined by joining this solution with the interior solution at $r=R$ (see below). Following the matching procedure outlined in Ref. 15. the low-energy $s$-wave scattering matrix is found as $S_{0}=$ $\tilde{S}_{0} e^{2 i \delta_{C}}$, where

$$
\tilde{S}_{0} \equiv e^{2 i \delta}=\frac{(\beta / \alpha)-i \varepsilon r_{s} C^{2}}{(\beta / \alpha)+i \varepsilon r_{s} C^{2}}
$$

is the short-range part of the scattering matrix, and $C^{2}=$ $2 \pi \varepsilon r_{s} /\left[1-\exp \left(-2 \pi \varepsilon r_{s}\right)\right]$ is a function that characterizes the long-range Coulomb tail of the potential 15$]$.

In general, resonances can be found as poles of the $S$ matrix in the complex energy plane at $\varepsilon=\varepsilon_{n}-i \gamma_{n} / 2$ [2]. On the real energy axis they correspond to the points where the short-range phaseshift $\delta$ passes through $\pi(n-$ $1 / 2)$. At these energies $\tilde{S}_{0}=-1$, which requires $\beta / \alpha=0$. This means that near the resonances one can use

$$
\beta / \alpha \simeq(\beta / \alpha)^{\prime}\left(\varepsilon-\varepsilon_{n}\right),
$$

where the prime denotes the derivative with respect to $\varepsilon$ taken at the resonance. Using this expression and looking 
for the poles of $\tilde{S}_{0}$, Eq. (21), gives the resonance width

$$
\gamma_{n}=2 \frac{r_{s} \varepsilon_{n} C^{2}}{(\beta / \alpha)^{\prime}}
$$

To find the ratio $\beta / \alpha$ and $(\beta / \alpha)^{\prime}$ we match the logarithmic derivatives of the interior and exterior wavefunctions at the boundary. Using the relation between the solutions $\psi(r)$ and $\phi(r)$ (Sec. III) for the interior metric, Eq. (8), the logarithmic derivative (evaluated at the boundary) is

$$
\begin{aligned}
\left.\frac{\psi^{\prime}(r)}{\psi(r)}\right|_{R-} & =\frac{1}{R}+\left.\frac{\phi^{\prime}(r)}{\phi(r)}\right|_{R-} \\
& =\frac{3}{R}-\frac{1}{R}\left(\frac{9 \epsilon}{2}\right)^{3} \tan \left[\frac{\pi \epsilon}{\sqrt{2 \xi}}-\frac{1}{3}\left(\frac{9 \epsilon}{2}\right)^{3}\right],
\end{aligned}
$$

where we used Eq. (16) to obtain the last line.

The exterior logarithmic derivative at the boundary for $r_{s} \rightarrow 8 / 9 R$ is found from Eq. (20) as

$$
\left.\frac{\psi^{\prime}(r)}{\psi(r)}\right|_{R+}=\frac{8 \alpha}{R(\beta-\alpha \ln 9)} .
$$

Setting the logarithmic derivatives from Eq. (24) and Eq. (25) equal, gives

$$
\frac{\beta}{\alpha}=\frac{8}{R\left[\psi^{\prime}(r) / \psi(r)\right]_{R-}}+\ln 9 .
$$

When finding $(\beta / \alpha)^{\prime}$ to estimate $\gamma_{n}$ from Eqn. (23), we can use the fact that at (or very near to) the resonance, $\beta / \alpha=0$. This allows one to simplify the answer and obtain

$$
\gamma_{n}=B \sqrt{\xi} \varepsilon_{n}^{4} R^{3}
$$

with the constant $B$ given approximately by $B=2.74$.

Thus we see that similar to the resonance energies $\varepsilon_{n}$, Eq. (19), the resonance widths also decrease with $\xi$, i.e., as the metric singularity is appoached. Taking Eq. (19) into account we see from Eq. (27) that for a fixed resonance number $n, \gamma_{n} \propto \xi^{5 / 2}$. This dependence is shown on the inset of Fig. 4 .

The above derivation of the width underestimates the width (through the value of $B$ ) by a factor $r_{2}^{2} / R^{2} \sim 0.1$, since the correct wavefunction does not decrease through region III as quickly as that given by Eq. (18) (see Fig. 22). Restoring this factor and evaluating $B$ numerically gives

$$
\gamma_{n} \simeq 28 \sqrt{\xi} \varepsilon_{n}^{4} R^{3}
$$

in good agreement with the numerical calculation, as shown by Fig. 4.

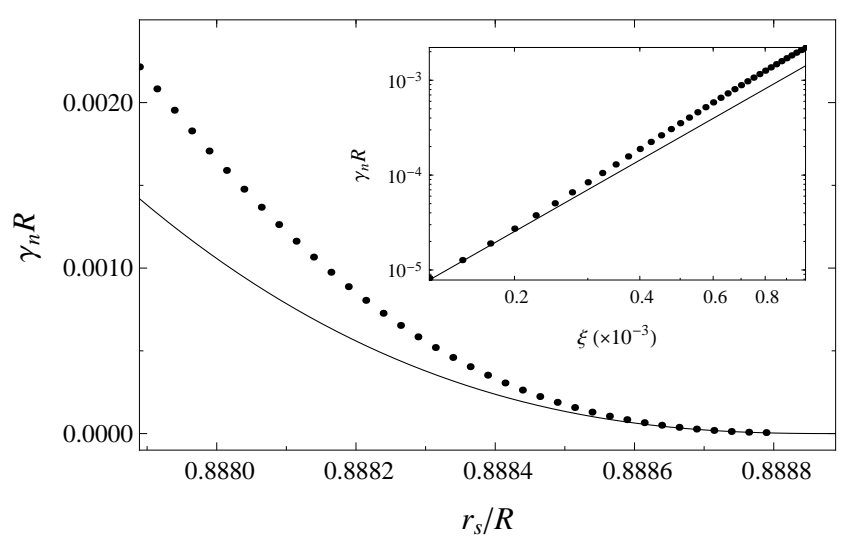

FIG. 4: Width of the $n=4$ resonance found numerically by fitting the phaseshift $\delta$ (solid circles), and estimated from Eqs. (19) and (28) (solid line), as a function of $r_{s} / R$. The inset shows the same data as a function of $\xi$ on a double logarithmic scale.

\section{ABSORPTION CROSS SECTION}

In the limit $r_{s} \rightarrow 8 R / 9$ the short-range phaseshift and the energy density of the resonances tend to infinity. Hence, neither $\delta$, nor the resonance energies and widths, $\varepsilon_{n}$ are $\gamma_{n}$ (which tends to zero) retain much physical meaning. However, as in the near-black-hole metric case 1], the cross section for capture into these resonances, i.e., the effective absorption cross section, is well defined.

Equation (19) shows the level spacing between the resonances is given by

$$
D \equiv \varepsilon_{n+1}-\varepsilon_{n}=\sqrt{2 \xi} / R
$$

Assuming a finite energy resolution $\Delta \varepsilon \gg D$ and given that $\gamma_{n} \ll D$, one can introduce the cross section for capture of the particle into these long-lived states. According to the optical-model considerations [2], this $a b$ sorption cross section is given by

$$
\bar{\sigma}_{a}^{(\mathrm{opt})}=\frac{2 \pi^{2}}{\varepsilon^{2}} \frac{\gamma_{n}}{D} .
$$

Substituting the expression for $\gamma_{n}$, Eq. (28), into the above expression yields

$$
\bar{\sigma}_{a}^{(\mathrm{opt})} \simeq 390 \varepsilon^{2} R^{4}
$$

Unlike the low-energy absorption cross section for the black holes, $\sigma_{a}=4 \pi r_{s}^{2}$, the cross section (31) vanishes at zero energy. However, for non-zero incident energies the capture capture cross section for massless particles is finite.

\section{CONCLUSIONS}

We have considered the problem of scattering of massless scalar particles from a spherically symmetric con- 
stant density fluid sphere described by the Schwarzschild interior metric. We find that despite the black hole limit being unattainable for such metric, a dense spectrum of narrow resonances emerges in the limit $r_{s} \rightarrow 8 R / 9$ in which a singularity develops in the metric at the origin.
This phenomenon gives rise to a nonzero capture cross section of massless particles for nonzero energis. This implies that an object that is not a black hole may gravitationally absorb particles making it appear black holelike, albeit with a different capture cross section.
[1] V. V. Flambaum,G. H. Gossel and G. F. Gribakin, Phys. Rev. D 85, 084027 (2012).

[2] L. D. Landau and E. M. Lifshitz, Quantum Mechanics, 3rd ed. (Butterworth-Heinemann, Oxford, 1977).

[3] W. G. Unruh, Phys. Rev. D 14, 3251 (1976); Thesis, Princeton Univ., 1971 (unpublished).

[4] R. A. Matzner, J. Math. Phys. 9, 163 (1968).

[5] A. A. Starobinskii, Zh. Eksp. Teor. Fiz. 64, 48 (1973) [Sov. Phys. JETP 37, 28 (1973)].

[6] N. Sanchez, Phys. Rev. D 18, 1030 (1978).

[7] S. R. Das, G. Gibbons, and S. D. Mathur, Phys. Rev. Lett. 78, 417 (1997).

[8] L. C. B. Crispino, S. R. Dolan, and E. S. Oliveira, Phys. Rev. Lett. 102, 231103 (2009).
[9] Y. Décanini, G. Esposito-Farèse, A. Folacci, Phys. Rev. D 83, 044032 (2011).

[10] K. Schwarzschild, Sitzungsber. Preuss. Akad. Wiss. 424 (1916).

[11] G. H. Gossel, J. C. Berengut, and V. V. Flambaum, Gen. Rel. Gravit. 43, 2673 (2011).

[12] M. Soffel, B. Müller, and W. Greiner, J. Phys. A 10, 551 (1977).

[13] H. A. Buchdahl, Phys. Rev. 116, 1027 (1959) .

[14] Mathematica, Version 7.0 (Wolfram Research, Inc., Champaign, IL, 2008).

[15] M. Yu. Kuchiev and V. V. Flambaum, Phys. Rev. D 70, 044022 (2004). 